\title{
Unusual Presentation of a Metastatic Tumor to the Temporal Bone: Severe Otalgia and Facial Paralysis
}

\author{
Sung Ho Choi, II-Seok Park, Young Bok Kim, and Seok Min Hong \\ Department of Otorhinolaryngology-Head and Neck Surgery, Hallym University, Dongtan Sacred Heart Hospital, \\ Hwaseong, Korea
}

Received May 22, 2013

Revised December 17, 2013

Accepted January 5, 2014

Address for correspondence

Seok Min Hong, MD

Department of Otorhinolaryngology-

Head and Neck Surgery,

Dongtan Sacred Heart Hospital,

7 Keunjaebong-gil,

Hwaseong 445-907, Korea

Tel +82-31-8086-2670

Fax $+82-31-8086-2681$

E-mail thecell@medimail.co.kr
Metastatic temporal bone tumors are rare diseases and they are usually clinically asymptomatic, so it is difficult to diagnose them. Breasts are the most common sites of temporal bone metastasis. Tumors of lung, kidney, gastrointestinal tract, prostate gland, larynx and thyroid gland are the other sites. The pathogenesis of the temporal bone is most commonly related to the hematogenous route. We present the case of a 78-year-old man with facial paralysis combined with severe otalgia. This patient was initially diagnosed with Bell's palsy. However, based on the radiologic findings, the patient was diagnosed with lung cancer with temporal bone metastasis.

Korean J Audiol 2014;18(1):34-37

\section{Introduction}

Metastatic temporal tumor is an uncommon disease, and it is difficult to be diagnosed because it is mostly shown with no clinical symptoms. ${ }^{1-5)}$ The most common origin of the metastasis is breast cancer, followed by lung, kidney, gastrointestinal tract, prostate, larynx and thyroid gland cancers. The most frequently detected metastatic pathway is the hematogenous pathway and the most common histological type is known to be an adenocarcinoma. ${ }^{3-5)}$ More and more cases of metastatic temporal bone tumors are discovered as the diagnostic techniques were recently developed and the life expectancy of the cancer patients is increased. In our country were reported 2 cases of prostate cancer and single cases of lung cancer, liver cancer, breast cancer and cancer of unknown origin. ${ }^{3,46-8)}$ Hereby, we report the case of a patient who was recently hospitalized with severe otalgia and facial paralysis, which was diagnosed as metastatic temporal bone tumor.

This is an Open Access article distributed under the terms of the Creative Commons Attribution Non-Commercial License (http://creativecommons. org/licenses/by-nc/3.0/) which permits unrestricted non-commercial use, distribution, and reproduction in any medium, provided the original work is properly cited.

\section{Case Report}

A 78 year-old man was admitted to the outpatient clinic of our department of otolaryngology with complaints of sudden pain of left auricle and ear canal. The patient also showed facial paralysis on left side (House-Brackmann grade III). He didn't have any underlying disease and showed a social history of smoking with 60 packs years and no history of alcohol intake. According to the physical examination, there was found no dermatological lesion such as rash, vesicle or crust near the ear drum, external auditory canal and near the external ear. The patient showed a sensorineural hearing loss of high frequency in both ears on pure tone audiometry. The hearing ability showed no worsening pattern as the symptoms were developed. The pain that was shown in this patient was of great severity compared to that generally shown in Bell's palsy, and he complained of a stronger, deep pain in the external auditory canal rather than in the areas near the auricles. Accordingly, beside to Bell's palsy, we considered an atypical type of Ramsay-Hunt syndrome that doesn't show dermatological lesions, as our possible differential diagnosis. We started with high doses of steroid (prednisolone, $60 \mathrm{mg} /$ day) and antiviral drug (Acylovir $1800 \mathrm{mg} /$ day) after the patient was hospitalized. Even 
though it's rare to present severe otalgia from primary or metastatic tumors of the skull base, we performed a CT scan of the temporal bone to rule out these possibilities. The findings from the CT scan showed a bony destruction of the temporal bone of the left, anterior attic and sphenoid bone (Fig. 1). Consequently, we could assume that the facial paralysis occurred due to damages of the first geniculate ganglion of facial nerve. We performed a MRI of the temporal bone in order to find out the etiology of the bony destruction of temporal bone and we observed a neoplastic lesion that is invading the base of the skull and left sphenoid bone. It was observed that the neoplastic lesion invaded the middle cranial fossa, temporalis muscle, left side of the foramen ovale and the left cavernous sinus and it could be diagnosed as a metastatic tumor of the temporal bone (Fig. 2). On the 8th day after hospitalization, we performed a positron emission tomography-computed tomography (PET-CT) scanning in order to confirm the presence of other metastasis to other organs and the existence of an originating region of the metastatic tumor. The PET-CT scan showed a high fluorodeoxyglucose uptake in the left middle crani- al fossa, both ribs, left scapula and the left adrenal gland. We also observed a lesion of $3 \mathrm{~cm}$ in the upper lobe of the right lung (Fig. 3) and tried to perform a biopsy on the upper lobe of right lung in suspicion of lung cancer with multiple areas of metastasis. However, it was not allowed as the guardians of the patient didn't want any invasive examinations or treatment, considering the patient's age and recovery rate after the treatment. Currently, the patient is under conservative treatment including pain management and he's under observation for 1 year so far.

\section{Discussion}

Facial paralysis of sudden onset is most commonly caused by Bell's palsy. ${ }^{1,2)}$ The etiologies of Bell's palsy is not wellknown, however, it's known to response well to steroid and anti-viral agents with good prognosis. ${ }^{1,2)}$ The second most common cause for the disorder is an infection. The infection may be an acute or chronic otitis media, infection with Human Immunodeficiency Virus, Epstein Barr Virus, Cytomegalovirus

Fig. 1. A: Non-enhanced temporal bone CT shows bony destruction in left middle skull base or lesser wing of left sphenoid bone (arrow). B: The enlarged picture shows suggested of destructive malignant tumor in left middle skull base (arrow).
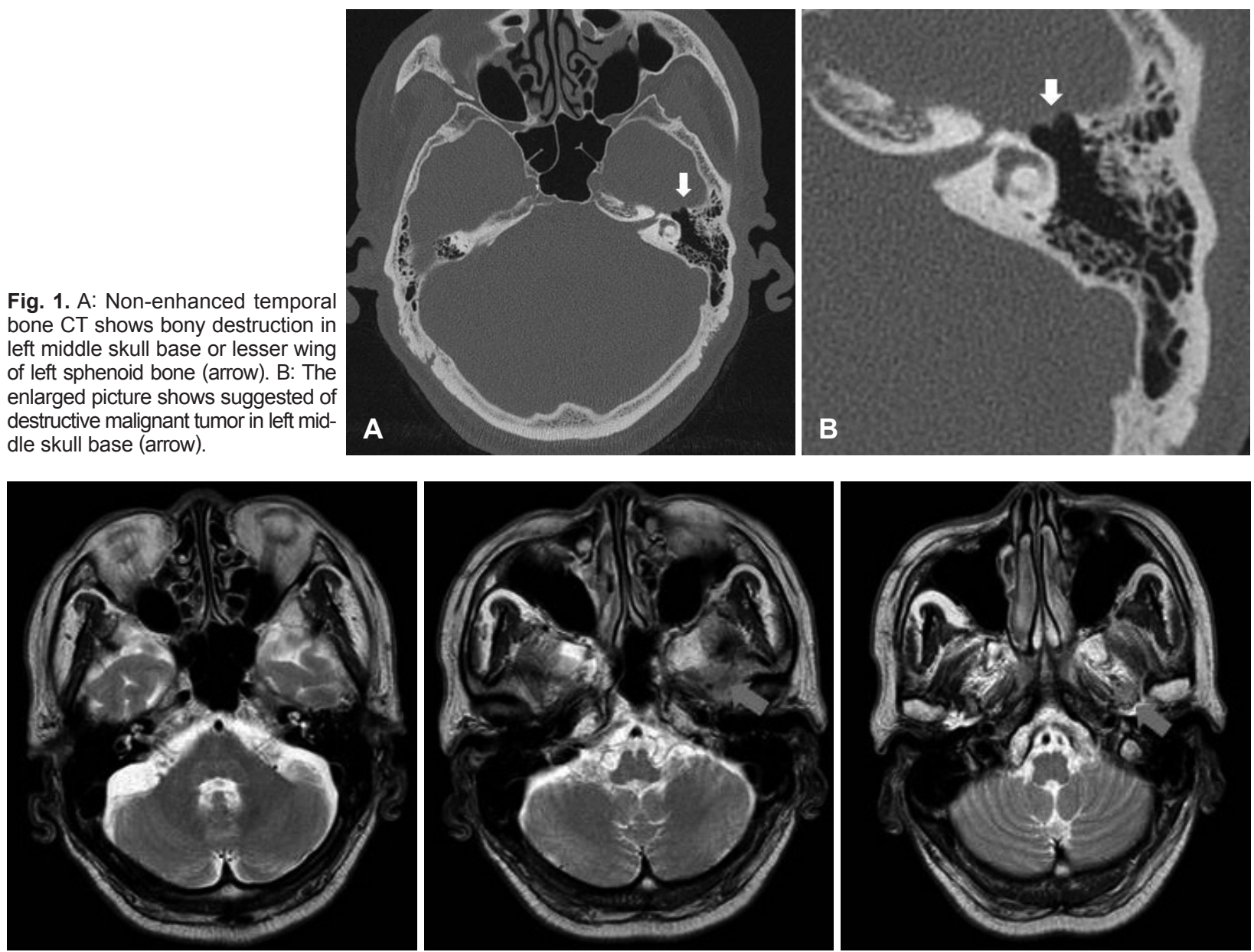

Fig. 2. Temporal bone MRI of the patient. A series of pictures. Low signal intensity mass lesion on T2-weighted image and involves left middle skull base. 

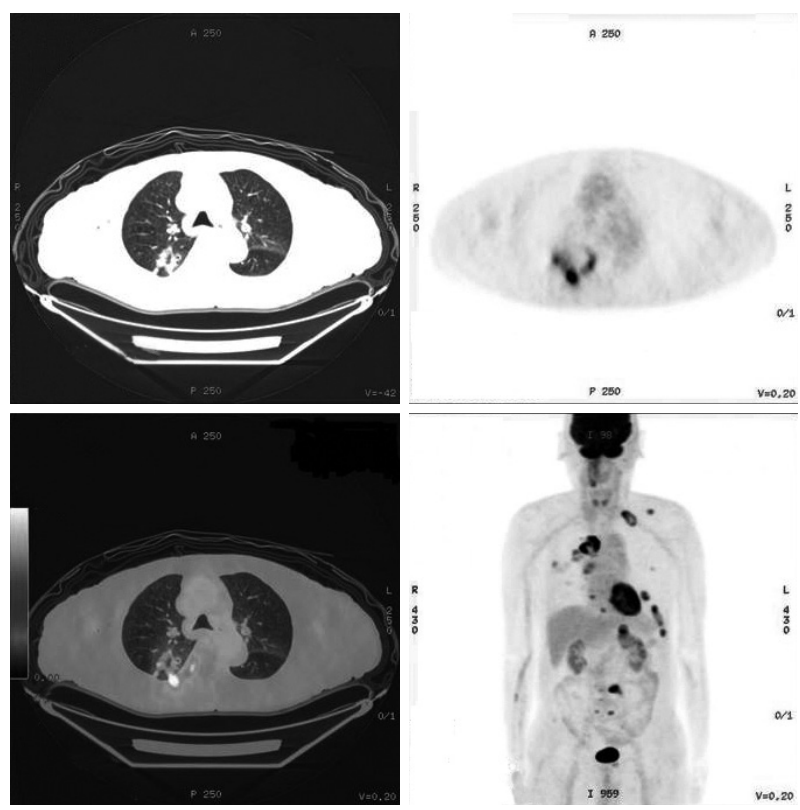

Fig. 3. PET-CT shows $3.4 \mathrm{~cm}$ sized cavitary lesion with moderate increased fluorodeoxyglucose (FDG) uptake in right upper lung lesion. Enlarged lymph nodes with FDG uptake in right interlobar and right hillar area. Multiple bony lesions with increased FDG uptake in bilateral ribs, left scapula, sternum, $L 4, S 1$, left middle cranial fossa. Left adrenal mass with increased FDG uptake. PET$\mathrm{CT}$ : positron emission tomography-computed tomography.

herpes viruses and Varicella Zoster Virus. ${ }^{2)}$ The facial paralysis due to brain tumor occurs in less than $5 \%$ of cases and it is rarely reported. The brain tumor tends to progress slowly and in case of a facial paralysis that slowly worsened or poorly responds to treatments such as with steroids or antiviral agents we can assume a facial paralysis occurred due to a brain tumor. ${ }^{1)}$

The otalgia and facial paralysis occurred 6 days in advance, however, we cannot be sure about the progression of facial paralysis as the patient was old and his chief complaint was mostly the deep ear pain instead of facial paralysis.

Most metastatic temporal tumor tends to be asymptomatic and the most commonly detected symptom is hearing loss. Other symptoms include otalgia, otorrhea, edema around the auricle, tinnitus, vertigo and facial paralysis. However, such symptoms can be present in otitis media and mastoiditis as well, and it is difficult to assume the symptoms to be characteristic of metastatic temporal tumor. Since most of the cases tend to be symptomless, we assume the actual prevalence of the disease to be higher than what's reported as it is commonly not diagnosed. ${ }^{3-5)}$ Our patient complained of much severe otalgia compared to that observed in patients with general facial paralysis. For this reason, we initially thought of an atypical Ramsay-Hunt syndrome. In retrospect, we could assume that this pain was mainly due to the compression on auriculotemporal nerve, a branch of mandibular nerve that passes through the foramen ovale by the metastatic tumor.
The spreading pathway of metastatic temporal tumor can occur due to several ways: firstly, a hematogenous spreading of primary origin of breasts, kidney or respiratory tract to bone marrow tissue of temporal bone. Secondly, it may be directly spreading through surrounding tissues such as nasopharynx, oropharynx, hypopharynx and parotid gland. Thirdly, tumor cells may spread through cerebrospinal fluid from intracranial tumor. Fourthly, it may spread through nearby meninges. Lastly, it may spread through lymphatic fluid. ${ }^{5)}$

Radiological examination is essential for the diagnosis of such disease, and any patterns of destruction or calcification can be observed with the help of CT scan of temporal bone. The osteolytic pattern that is observed frequently may be present in choleasteatoma also, so it's difficult to diagnose differentially. ${ }^{5)}$ The MRI images allows a clearer visualization of soft tissues with better diagnostic value, however, it has cost issues. In order to discover the primary origin of tumor, PET-CT may be quite useful. For confirmation of diagnosis, though biopsy of lesion of temporal bone is necessary. It is difficult to obtain the sufficient amount of tissue, and the procedure may lead to damages of surrounding tissues. For this reason, this procedure has many difficulties. ${ }^{3-5)}$

Generally, radiological examination could be chosen depending on the location of the lesion and clinical feature in case of facial paralysis. If the facial paralysis is considered to be due to lesions that are limited to pontine nuclei or internal acoustic canal, we perform a contrast-enhanced MRI first. On the other hand, if the lesions are thought to be limited to the mastoid, ear drum or labyrinthine segment, then we generally perform a high-resolution CT scan of the temporal bone. A contrast-enhanced CT scan might be thought to be recommended if the location of origin of facial paralysis is unknown.

The treatment generally initiates conservatively and physicians may consider operation, chemotherapy and radiotherapy depending on the patient's status. The prognosis of the patients is related to the progression of the primary site or whether the patient was treated or not. The 2-year survival rate was reported to be $50-80 \%$ in the patients who received operation and radiotherapy for their primary cancer and was reported to be as poor as $0-40 \%$ in the patients of terminal stage, even though they have received chemotherapy and radiotherapy before. ${ }^{9,10)}$

The metastatic tumor of the temporal bone may not be a common disorder, however, it can be suspected in cases that are accompanied by slowly-progressed hearing loss, facial paralysis and poor response to the treatment. In our patient's case, a severe otalgia accompanying the facial paralysis was an important clue for the diagnosis of metastatic temporal tumor. Finally, we conclude that early efforts by thoroughly observ- 
ing the symptoms of the patient are necessary to diagnose and treat the metastatic temporal tumor.

\section{REFERENCES}

1) Boahene DO, Olsen KD, Driscoll C, Lewis JE, McDonald TJ. Facial nerve paralysis secondary to occult malignant neoplasms. Otolaryngol Head Neck Surg 2004;130:459-65.

2) Yildiz O, Buyuktas D, Ekiz E, Selcukbiricik F, Papila I, Papila C. Facial nerve palsy: an unusual presenting feature of small cell lung cancer. Case Rep Oncol 2011;4:35-8.

3) Shim HJ, Jun BS, Park SC, Kim SH. Metastatic adenocarcinoma of the temporal bone presenting as facial paralysis. Korean J Otolaryngol-Head Neck Surg 2006;49:1130-3.

4) Lee JM, Jung KT, Soh UK, Han JH. A case of facial paralysis caused by metastatic breast carcinoma. J Clinical Otolaryngol 2012;23:105-9.

5) Lan MY, Shiao AS, Li WY. Facial paralysis caused by metastasis of breast carcinoma to the temporal bone. J Chin Med Assoc 2004;67: 587-90.

6) Choi W, Kim YS, Shin YW, Kwon KS, Lee DH, Cho HG, et al. A case of metastatic hepatocellular carcinoma presented as isolated facial palsy. Korean J Gastroenterol 1999;34:406-10.

7) Chang KH, Seo JH, Choi SH, Park YS. A case of metastatic adenocarcinoma of unknown primary origin involving the temporal bone. Korean J Otorhinolaryngol-Head Neck Surg 2008;51:850-3.

8) Heo KW, Kim JS, Jung SJ, Bae SK. Delayed diagnosis of metastatic prostatic adenocarcinoma in temporal bone. Korean J Otorhinolaryngol-Head Neck Surg 2010;53:179-83.

9) Moffat DA, Wagstaff SA, Hardy DG. The outcome of radical surgery and postoperative radiotherapy for squamous carcinoma of the temporal bone. Laryngoscope 2005;115:341-7.

10) Moody SA, Hirsch BE, Myers EN. Squamous cell carcinoma of the external auditory canal: an evaluation of a staging system. Am J Otol 2000;21:582-8. 